\title{
PRESSURIZED PERFUSION SYSTEM FOR OBTAINING COMPLETEIY ACELLULAR PULMONARY VALVE SGAFFOLDS FOR TISSUE ENGINEERING
}

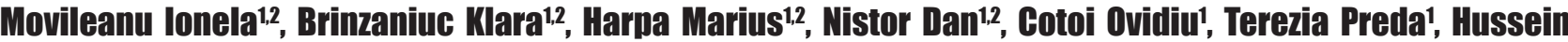 Hussam Al12, Moldovan Oana', Man Adrian', Harceaga Lucian', Sierad Leslie ${ }^{3}$, Simionescu Dan 1,4}

\author{
${ }^{1}$ University of Medicine and Pharmacy, Science and Technology, George Emil Palade, Targu Mures, Romania \\ ${ }^{2}$ Institute of Cardiovascular Diseases and Transplant, Targu Mures, Romania \\ ${ }^{3}$ Aptus Bioreactors, LLC, Clemson, SC, USA \\ ${ }^{4}$ Clemson University, South Carolina, USA
}

\begin{abstract}
Introduction. Xenogeneic tissues decellularization represents the obtaining process of extracellular matrix derived scaffolds. Most antigens being cell based, non-immunogenicity is obtained by cells removal. Scaffolds are temporary structures with biologic and mechanical role. Scaffolds, stem cells and bioreactors represent premise of regenerative medicine, aiming towards the ideal valvular substitute. In previous studies, we decellularized pulmonary valves root by immersion histology revealing cellular residue, requiring a more efficient approach. We hypothesized that immersion is insufficient and thus a pressure gradient was added. Material and Method. This is part of a grant approved by the UMFTS. Eleven porcine pulmonary valves were included in the study: $n=6$ underwent immersion decellularization and $n=5$ were cyclically perfused with a 20-25 $\mathrm{mmHg}$ pressure gradient during a 10-day protocol. The acellular valves obtained underwent a quality control using DAPI (4',6-diamidino-2-phenylindol) nuclear staining, histological Haematoxylin-Eosin, DNA extraction and quantification, harvested from different structural levels: arterial wall, sinus, cusp. Results. Histological assessments highlighted integrity of extracellular matrix in both groups and overall cells absence at the different levels of valvular structures analyzed. Immersion decellularized valves exhibited DAPI positive structures identified as potential residual nucleic material. Comparatively, the perfusion decellularized valves, lacked in those structures, result confirmed by DNA extraction and quantitation procedure. Conclusions. Perfusion decellularization represents a feasible approach to obtain acellular cardiac valvular scaffolds derived from the extracellular matrix, being superior to immersion decellularization method. Their nonimmunogenic potential is underlined by total absence of nuclei. The process is fast, allowing production of an abundant number of valvular biomaterials in a short time.

Keywords: heart valve, acellular, decellularization, regenerative medicine
\end{abstract}

\section{Introduction}

Regenerative medicine, a new research field, represents the laboratory based strategy through which cardiologists, cardiac surgeons and scientists aspire to manufacture a living heart valve substitute. Valvular heart disease represents a major morbidity and mortality among elderly patients. Since the improved treatment for rheumatic fever it was noted an increase in the degenerative etiology of the valvular diseases (1). Their treatment is exclusively interventional or surgical, tending towards less invasive procedures, replacing the valves by transcutaneous (transcatheter) approach $(2,3,4)$. Medical treatment represents an option only as a heart failure symptoms releaser. Surgical treatment is represented by valve reconstruction or vale replacement with either a biological or a mechanical prosthesis, decision made by a multidisciplinary team with the patient (5). Although when possible, reconstruction is preferred in order to preserve the valvular apparatus and the ventricular function (6) studies reveal an increasing number of valvular replacement $(7,8)$.

Standard heart valve prostheses are represented by the mechanical valves and by the chemically cross-linked biological valves. Although their usage represented a major step in 
the improvement of life quality in patients with valvular heart disease, their shortcomings make them suboptimal (9). Mechanical valves require lifelong anticoagulation therapy with anti-vitamin $\mathrm{K}$ agents who needs additional periodically testing of the anticoagulation efficiency by dosing the International Normalized Ratio (INR) individually adapted to the prosthesis type and patient's risk factors (10). Regarding biological prosthesis, their downside is represented by the limited lifespan, requiring re-intervention either surgical or interventional and replacement due to calcification and degeneration (11).

The classic tissue engineering paradigm is based on the synergic usage of scaffold, different cells and varied stimuli(12). Scaffolds are temporary physical support for the cells, until they start degrading it. Simultaneously with the destruction process, the cells produce their own extracellular matrix, replacing the scaffold, and obtaining a new living tissue. By their origin, scaffolds are divided into synthetic, composed of polymers(13-16) and biologic, derived from tissue's extracellular matrix (ECM) $(17,18)$. Biologic scaffolds are preferred over the synthetic ones because: 1) Hydrolytic degradation of some polymers generates toxic compounds, with detrimental effect on cells development(19) and 2) biologic scaffolds offer $3 \mathrm{D}$ architectural and biochemical environments conducive to cell repopulation and growth.

In our study we aimed to manufacture a closely to ideal tissue engineered heart valve (TEHV) based on porcine pulmonary valves by using two different decellularization processes. Decellularization is a relative common procedure in regenerative medicine domain, that by using a succession of detergents, enzymes and alcohols, varied biologic scaffolds derived from tissue`s ECM are obtained (20-23).

\section{Material and Methods}

This research is part of a grant that was approved by the Ethics Committee of University of Medicine and Pharmacy Science and Technology, George Emil Palade from Targu Mures with no 131/2016.

\section{Porcine hearts procurement and} pulmonary valves harvesting

The porcine hearts were provided by a local abattoir form animals weighing between 80 and $100 \mathrm{~kg}$. After their fast transportation on ice to the Tissue Engineering and Regenerative Medicine Laboratory, the pulmonary valve was carefully dissected and isolated. The isolation protocol was adapted depending on which study group they followed to be integrated.

For the perfusion decellularization group, the valves needed to be fixated in plastic 3D printed rings through a trimmed portion of the sub-valvular myocardium. By using this fixation it was possible toexpose the valves to perfusion and obtain a pressure gradient between the inner and the outer compartment that circulated the decellularization solutions. The fitting component of the pulmonary valve was represented by a circular 3-4 cm wide flap of myocardial tissue. Taking into consideration the fact that during the decellularization protocol, the myocardium was getting even thinner, a spring was added in order to seal the two spaces, and the pressures could be held at a constant level.Regarding the length of the arterial component, the pulmonary trunk was sectioned beneath the bifurcation (Figure 1).

In the immersion group, the preserved myocardial tissue was reduced to the minimum that kept the integrity of the valvular annulus considering the fact that leaflets bases are implanted directly on the myocardium. The retained part of the arterial was about two $\mathrm{cm}$ above the three commissures (Figure 1).

Afterward, the freshly harvested pulmonary valves were cleaned of any residues and multiple times rinsed with water.

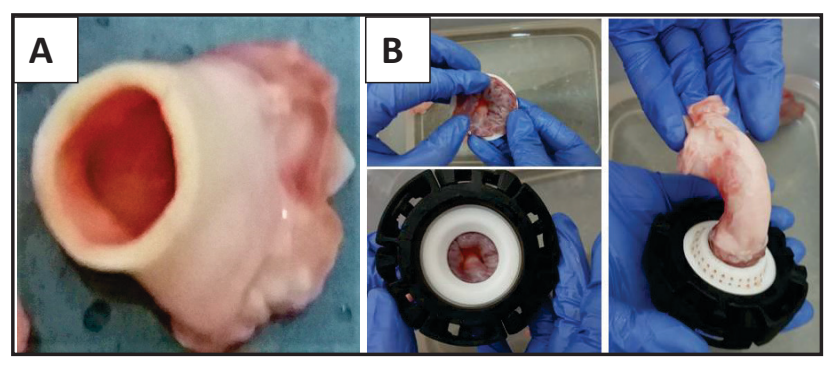

Figure 1 - General aspects of the isolated porcine pulmonary arteries prepared for: - A-the immersion protocol and $B$ - perfusion protocol 


\section{Immersion decellularized pulmonary valves group}

Consisting in a five day lasting protocol, the porcine pulmonary valves were successively immersed in varied decellularization solution mixes and placed on an orbital shaker, aiming to increment the procedure effect. Subsequently to the cleaning process, the valves were firstly immersed in $0.02 \%$ Sodium Azide in $\mathrm{H} 2 \mathrm{O}$ and placed on the orbital shakerat the lowest speed that ensures movement of the valves in the solutions, at room temperature (250 Celsius) for 24 hours, to induce cells destructioninduced by the osmotic gradients. The next day the valves were abundantly water rinsed and exposed to $70 \%$ ethyl alcohol for twenty minutesextract cell lipid components. This step was followed by distilled water rinsing and one hour incubation in $0.05 \mathrm{M}$ Sodium Hydroxide. Thereafter the washing out the sodium hydroxide with water the decellularization solution $(0.05 \%$ SDS Sodium Dodecyl Sulfate, $0.5 \%$ TRITON X-100, $0.5 \%$ DOC - Deoxycholic Acid, Sodium salt, $0.2 \%$ EDTA - Ethylenediaminetetra-acetic acid, $10 \mathrm{mM}$ TRIS- Hydroxymethyl, $\mathrm{pH}$ to $7.4 \pm 0.05$ ), was introduced and agitated continuously for 24 hours at the laboratory room temperature.

The decellularization solution was cleared through multiple distilled water rinsing (five times) followed by three successive immersions for five minute in water and an abundant water rinsing lasting until the detergent foam was eliminated. This step proceeded to an immersion for twenty minutes in $70 \%$ ethylic alcohol and three times distilled water rinsing. The alcohol treatment of the valves was rehydrated with two hours incubation in distilled water. Next, valves were placed in nucleic acid degrading enzymes - Ribonuclease (RN-ase)/Deoxyribobuclease (DNase)( 360mU/mL RNase+ $360 \mathrm{mU} / \mathrm{mL}$ DN-ase in PBS 1x (Phosphate-buffered saline), $1.015 \mathrm{~g}$ magnesium chloride - $\mathrm{MgCl} 2$ )at 370 Celsius, on a shaker, for 24 hours.

In the fourth day of the protocol, the enzymes were removed; the valves were rinsed three times with distilled water and placed in 70 $\%$ ethylic alcohol for three hours, on a shaker at room temperature. Sequential to this step, the whole valve manipulation was performed in sterile conditions: in a sterile hood, using sterile bottles, sterile solutions and tools. Using sterile distilled water the valve were rising once and following incubated for fifteen minutes in sterile distilled water on the shaker. Next the valves were placed in sterile PBS $1 x$ for half an hour and within a new BPS solution, overnight.

In the last day of the protocol, the valves were transferred to new sterile bottles and incubated for two hours in $0.2 \%$ peracetic acid in PBS 1x on the shaker at room temperature in order to sterilize them. The peracetic acid was cleared out with repetitive (three times) PBS immersions, each lasting for fifteen minutes. The immersion protocol was finished by individual placing of them in new sterile bottles in sterile PBS and 40 Celsius refrigeration.

\section{valves group \\ 3. Perfusion decellularized pulmonary}

The heart valve decellularization system was assembled as described before (24). It was designed in a manner that a maximum of five valves could be processed at a time (Figure 2). For this purpose, five pulmonary valves were harvested with an adequate length of sub-valvular myocardium. Next, the valves were fixed in the fitting rings at their base and the arterial side was clamped with an angled connector. The cocktail of decellularization solutions, each solution being prepared in a four liters quantity, was cyclically circulated by a peristaltic pomp. Before the starting of the pomp, the pressure transducers were calibrated to the zero value. The perfusion regimen consisted in alternative three minutes cyclically perfusion with 30 seconds break at a rate of $550 \mathrm{~mL} /$ minute in order to maintain a pressure gradient that allows an excursion of the walls between the on and break phases (average of $25 \mathrm{mmHg}$ ).

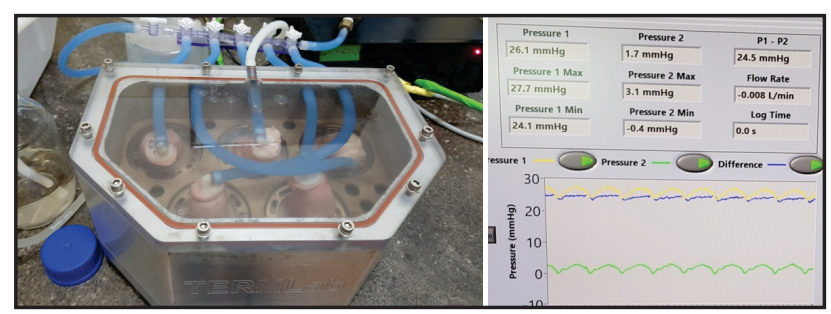

Figure 2 - General aspects of the perfusion decellularization system and the pressures revealed by the software. 
The protocol started the same as for the immersion protocol with $0.02 \%$ Sodium Azide circulated for 24 hours, at room temperature. After drainage of Sodium Azide, the system was filled with $0.05 \mathrm{M}$ Sodium hydroxide and left to action for one hour. The base was cleared out by distilled water rising followed by two hours perfusion. At the end of the day the adapted decellularization solution $(0.25 \%$ SDS, $0.5 \%$ TRITON X-100, 0.5\% DOC, 0.2\% EDTA, 50 $\mathrm{mM}$ TRISpH to $7.4 \pm 0.05$ ), was introduced and perfused for two days continuously when a new, fresh solution was introduced cycled also for a time-span of 48 hours.

Decellularization solutions were washed out with a two hours perfusion of distilled water, and pre-warmed at 370 Celsius DN-ase and $\mathrm{RN}$ ase enzymes wereintroduced and left to action for 48 hours. The perfusion was performed at this high temperature by placing the bottle containing the enzymes on a hot plate.

Clearance of enzymes was done with washing with BPS for two hours followed by two times perfusion of $70 \%$ ethyl alcohol each lasting two hours and overnight $0.02 \%$ Sodium Azide in 1x PBS for 48 hours. The finishing day of the protocol comprised the sterilization step with $0.2 \%$ peracetic acid performed by one hour perfusion regimen and one hour of shaker regimen in sterile bottles after the decellularization machine disassemble. With $1 \mathrm{x}$ BPS rising and an hour immersion on the orbital shaker ar room temperature, the acid was cleared away and the acellular valves were transferred in new sterile and labeled bottles to the refrigerator in 1x PBS.

control

4. Decellularization procedure quality

In order to assess the effects of the decellularization and their magnitude a series of test were performed regarding the sterility and the nuclei absence by using histology staining and DNA extraction.

In order to test each anatomical component of the valves, one valve was randomly picked as the group control and its three components were isolated and analyzed. analyzed. This will be referred to as the "test valve".Also, to check the quality of each valve, we collected small fragments from the wall of each valve.

\section{4.a. Sterility control}

Valve samples and their storage solution (PBS 1x) were cultivated on regular agar growth media and also on enriched growth media in an incubator at $370 \mathrm{C}$ for a time period of 72 hours, with sequent analysis and interpretation.

\section{4.b. Cell absence evaluation}

Integrity of the ECM and the absence of the nuclei were evaluated by using histological examinations. The first histology test performed was the nuclear staining DAPI - a fluorescent stainthat binds strongly to the DNA domains enriched in adenine-thymine bonds (25). Cryosections from the wall, the sinus and the leaflet of the test valve and wall sections form each of the decellularized valves were stained with DAPI and analyzed by fluorescence microscopy. Photographs were taken from DAPI stained sections using identical exposure times.

Samples for histology were fixed in formalin and embedded in paraffin. Sections of 5 um (micrometer) were cut on the microtome and thereafter stained with Hematoxylin and Eosin The microscope slides were then evaluated by opticalmicroscopy.

For DNA analysis, decellularized tissues, in parallel with fresh native tissues as controls, were weighed and DNA extraction and purification was performed on 11-15 mg tissue samples with a commercially available extraction kit (26) and quantified by Nano-Drop spectroscopy. DNA amounts were normalized to tissue weight. Electrophoresis of the purified nucleic material was performedonagarose gels, intercalated with Ethidium bromide.

\section{Results}

Eleven porcine pulmonary valves underwent the decellularization protocol. Through the procedures, there were no complications or incidents.

\section{Sterility control}

Microbiology tests revealed the sterility of the valves and their preservation PBS 1x.

\section{Histological determinations}

For both study groups, the DAPI staining revealed the architecture of the extracellular matrix with the consecrated features regarding its components: leaflet, valve sinus and arterial wall. 
The studied leaflets in the two study groups presented the collagen arrangement representing the fibrosa. Absence of the resident endothelial cells on the arterial and ventricular sides were noted also lack of cells inside the ECM.

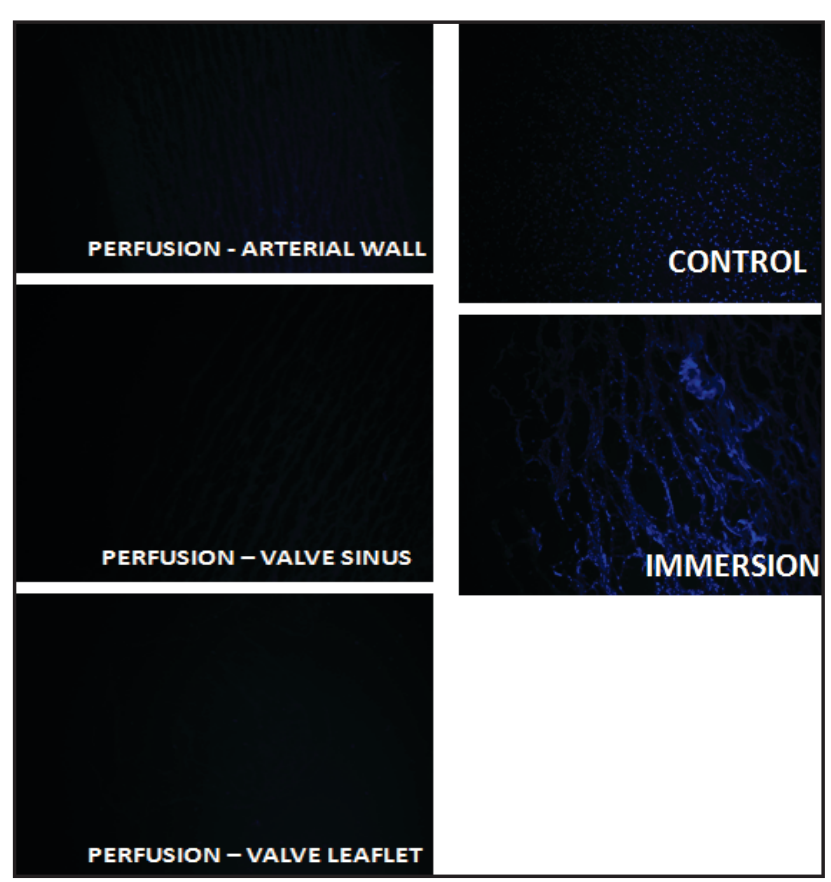

Figure 3-DAPI staining - comparison of the two decellularized groups with the control-A-perfusion decellularization, $B$ - immersion

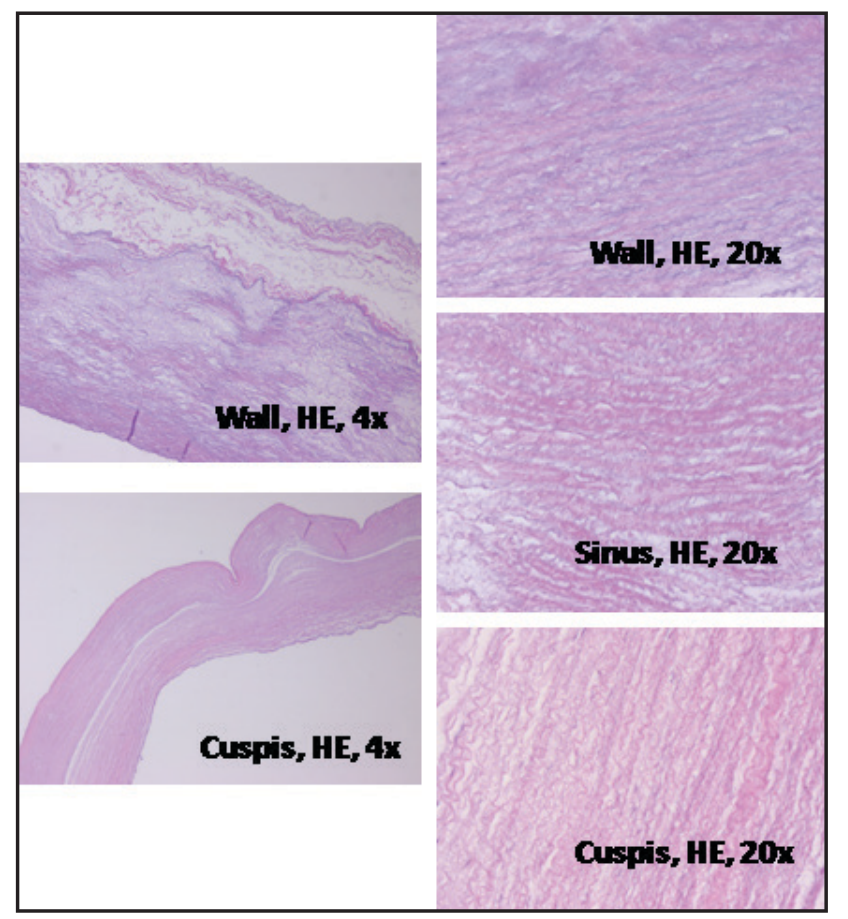

Figure 4 - Hematoxylin Eosin Stain optical microscopy aspects - perfusion decellularized valve - wall, sinus, cuspis - revealing the pink colored extracellular matrix and the absence of blue stained nuclei.
For the sinuses and the arterial walls, the three layer structure was identified-tunica intima, tunica media and tunica adventitia. Absence of the endothelial cells was observed in the intima layer. The major differences were noted in the tunica media of the immersion-decellularized porcine pulmonary valves presenting at the DAPI stainingexaminations, numerous conglomerates, suggesting nucleic and cellular remnants. These structures were absent in the valves form the perfusion group (Figure 3 and Figure 4).

\section{DNA material examination}

The DNA extraction and its intensity of absorbancemeasured with a spectrophotometer, from all anatomical structures of the control valve revealed the nucleic material purity and it wasreferred to the freshly harvested tissue in terms of weight (Table $1)$.

Table 1. DNA extraction results. *since DNA was extracted from about $15 \mathrm{mg}$ tissue and the detection limit of NanoDrop is about $2 \mathrm{ng} /$ sample, these values can be interpreted as $<0.13 \mathrm{ng} / \mathrm{mg}$ tissue.

\begin{tabular}{|l|r|r|}
\hline \multicolumn{1}{|c|}{ Tissue type } & $\begin{array}{c}\text { DNA } \\
\text { concentration } \\
\text { (ng/mg) }\end{array}$ & Purity \\
\hline $\begin{array}{l}\text { Porcine fresh pulmonary } \\
\text { leaflet }\end{array}$ & 8.5 & 1.778 \\
\hline $\begin{array}{l}\text { Porcine fresh pulmonary } \\
\text { arterial wall }\end{array}$ & 39 & 1.857 \\
\hline $\begin{array}{l}\text { Porcine fresh pulmonary } \\
\text { sinus }\end{array}$ & 72.5 & 1.859 \\
\hline $\begin{array}{l}\text { Porcine decellularized } \\
\text { pulmonary leaflet }\end{array}$ & $*$ Undetectable & Undetectable \\
\hline $\begin{array}{l}\text { Porcine decellularized } \\
\text { pulmonary arterial wall }\end{array}$ & Undetectable & Undetectable \\
\hline $\begin{array}{l}\text { Porcine decellularized } \\
\text { pulmonary sinus }\end{array}$ & Undetectable & Undetectable \\
\hline
\end{tabular}

Further quantitative evaluation of the DNA samples was performed by running them stained with ethidium bromideon an agarose gel electrophoresis that revealed absence of migration lines (Figure 5). 


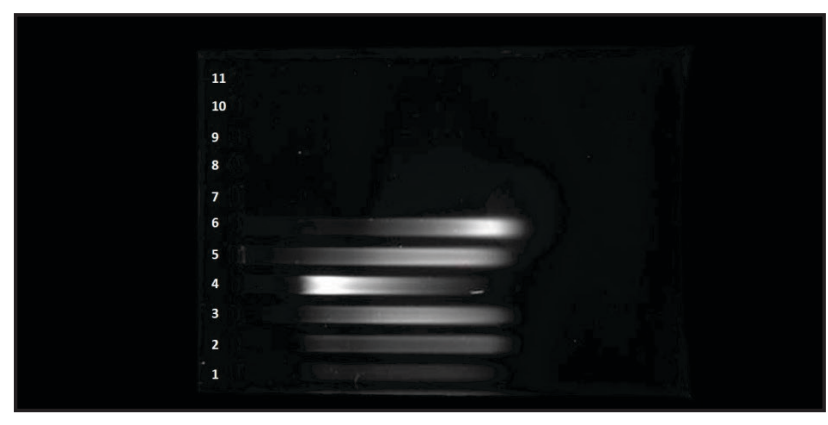

Figure 5 - Gel electrophoresis - DNAmigration lines. 1 to 6 lines-Fresh tissue.7-11 lines-decellularized tissue.

\section{DISEUSIONS}

When compared to the aortic valve, the pulmonary valve is generally considered a less complex valve; a recent search of the literature revealed limited data regarding this valvular structure. One explanation to this fact could be fact that the diseases involving the valvular structures belonging to the left side are more extensive due to the hemodynamic regimens they are cyclical exposed. An additional reason for the major attention span directed towards the aortic valve is its more complex in anatomic structures.

When it comes to the scientific research, the pulmonary valves could represent an initial step for studies, due to multiple factors: the ease of access - the valve is placed in the most superficial anatomic plan of the heart, the absence of the coronary ostia make them facile to handle and due to the pressure regimens the valvular prostheses wanted to be tested, are not exposed to aggressive hemodynamic conditions.

This paper comes as a follow-up to our prior studies where we used and described the perfusion decellularization for porcine aortic valves (24), confirming once again the importance of the physical component - pressure gradient in the decellularization procedure. In preliminary studies we have confirmed that acellular porcine pulmonary valves perform very well hemodynamically in vitro and in vivo; these results will be the subject of a separate publication (in preparation).

Regarding the pulmonary valve replacement, bio-prostheses are preferred due to the fact that mechanical prostheses in pulmonary position require assertive anticoagulation (27) and it was reported a slower degeneration rate when compared to the aortic position and the lack of thromboembolic risk (28). Pediatric pulmonary replacement procedure commonly presented in pediatric cardiology clinics - a study performed in Germany situated the pulmonary stenosis as the third congenital heart disease (29). This procedure is considered more challenging due to the fact that the implantation is performed in a growing organism.

At the moment there is an interest regarding decellularized pulmonary valve, studies entering the translational phase. The on-going ESPOIR trial targeting patients aged $21.3 \pm 14.4$, aims to compare decellularized homografts to the current alternatives - the bovine jugular vein conduit - Contegra conduit(30) and cryopreserved homografts, their initial results from registry data showed their superiority (31). The ARISE trial represents a further step, targeting the pediatric valve replacement using decellularized aortic homografts(32).

Although first results are enthusiastic, a possible shortcoming could be presented by the limited number of donors, as source for the heart valves. By using a xenografic source, an extensive number of valves could be obtained in varied sizes.

\section{STUDY LIMITATIONS}

The immersion decellularization group was not investigated regarding the DNA levels due to the fact thatDAPI screening revealed the presence of DNA and thus it was considered a deficient method. The visible incomplete decellularization, revealed by histology made the further investigations non-efficient.

Having in mind the fact that a more aggressive decellularization approach could produce harm to the ECM structure, further studies are needed in order to test the mechanical behavior of the decellularized valves.

\section{CONCLUSIONS}

Tissue decellularization procedure represents an appropriate process in order to obtain acellular heart valve ECM based scaffolds. In our study we demonstrated that by enclosing a 
pressure gradient to the protocol, a more optimum decellularization is achieved.

By applying this protocol, with sterile tissue and non-detectable DNA level at the procedure finale, their non-immunogenic profile is stated. Considering their xenogeneic origin and the protocol's limited extension, production of large number of bioengineered heart valve substitutes could provide off-shelf, individual tailored prostheses.

\section{CONFLICT OF INTEREST}

None to declare

\section{ACKNOWLEDGEMENT:}

This work was fundedby a grant from the Competitiveness Operational Programme2014-2020, Tissue engineering technologies for cardiac valve regeneration, VALVE-REGEN, idP_37_673, Mysmis code:103431, contract 50/05.09.2016.

\section{REFERENCES}

1. Coffey S, Cairns BJ, Iung B. The modern epidemiology of heart valve disease. Heart. 2016 Jan;102(1):75-85.

2. Cribier A. Development of transcatheter aortic valve implantation (TAVI): a 20year odyssey. Arch Cardiovasc Dis. 2012 Mar;105(3):146-52.

3. Cullen MW, Cabalka AK, Alli OO, Pislaru SV, Sorajja P, Nkomo VT, et al. Transvenous, antegrade Melody valve-invalve implantation for bioprosthetic mitral and tricuspid valve dysfunction: a case series in children and adults. JACC Cardiovasc Interv. 2013 Jun;6(6):598-605.

4. Schillinger W, Senges J. (TRAMI (Transcatheter Mitral Valve Interventions) register. The German mitral register). Herz. 2013 Aug;38(5):453-9.

5. Elwyn G, Frosch D, Thomson R, JosephWilliams N, Lloyd A, Kinnersley $P$, et al. Shared decision making: a model for clinical practice. J Gen Intern Med. 2012 Oct;27(10):1361-7.

6. Friedewald VE, Bonow RO, Borer JS,
Carabello BA, Kleine PP, Akins CW, et al. The Editor's Roundtable: cardiac valve surgery. Am J Cardiol. 2007 May 1;99(9):1269-78.

7. Mikos AG, Herring SW, Ochareon P, Elisseeff J, Lu HH, Kandel R, et al. Engineering complex tissues. Tissue Eng. 2006 Dec;12(12):3307-39.

8. Yacoub MH, Takkenberg JJ. Will heart valve tissue engineering change the world? Nat Clin Pract Cardiovasc Med. 2005 Feb;2(2):60-1.

9. van Geldorp MW, Eric Jamieson WR, Kappetein AP, Ye J, Fradet GJ, Eijkemans MJ, et al. Patient outcome after aortic valve replacement with a mechanical or biological prosthesis: weighing lifetime anticoagulantrelated event risk against reoperation risk. J Thorac Cardiovasc Surg. 2009 Apr;137(4):881-6, 6e1-5.

10. Baumgartner H, Falk V, Bax JJ, De Bonis M, Hamm C, Holm PJ, et al. 2017 ESC/ EACTS Guidelines for the management of valvular heart disease. Eur Heart J. 2017 Sep 21;38(36):2739-91.

11. Hoffmann G, Lutter G, Cremer J. Durability of bioprosthetic cardiac valves. Dtsch Arztebl Int. 2008 Feb;105(8):143-8.

12. Langer R, Vacanti JP. Tissue engineering. Science. 1993 May 14;260(5110):920-6.

13. Sodian R, Hoerstrup SP, Sperling JS, Daebritz S, Martin DP, Moran AM, et al. Early in vivo experience with tissue-engineered trileaflet heart valves. Circulation. 2000 Nov 7;102(19 Suppl 3):III22-9.

14. Shinoka T, Ma PX, Shum-Tim D, Breuer CK, Cusick RA, Zund G, et al. Tissueengineered heart valves. Autologous valve leaflet replacement study in a lamb model. Circulation. 1996 Nov 1;94(9 Suppl):II164-8.

15. Hoerstrup SP, Sodian R, Daebritz S, Wang J, Bacha EA, Martin DP, et al. Functional living trileaflet heart valves grown in vitro. Circulation. 2000 Nov 7;102(19 Suppl 3):Iii44-9.

16. Shinoka T, Breuer CK, Tanel RE, Zund G, Miura T, Ma PX, et al. Tissue engineering heart valves: valve leaflet replacement study in a lamb model. Ann Thorac Surg. 1995 Dec;60(6 Suppl):S513-6.

17. Badylak SF, Taylor D, Uygun K. Wholeorgan tissue engineering: decellularization 
and recellularization of three-dimensional matrix scaffolds. Annu Rev Biomed Eng. 2011 Aug 15;13:27-53.

18. Dahl SL, Koh J, Prabhakar V, Niklason LE. Decellularized native and engineered arterial scaffolds for transplantation. Cell Transplant. 2003;12(6):659-66.

19. Sung HJ, Meredith C, Johnson C, Galis ZS. The effect of scaffold degradation rate on three-dimensional cell growth and angiogenesis. Biomaterials. 2004 Nov;25(26):5735-42.

20. Schaner PJ, Martin ND, Tulenko TN, Shapiro IM, Tarola NA, Leichter RF, et al. Decellularized vein as a potential scaffold for vascular tissue engineering. J Vasc Surg. $2004 \mathrm{Jul} ; 40(1): 146-53$.

21. Kajbafzadeh AM, Javan-Farazmand N, Monajemzadeh M, Baghayee A. Determining the optimal decellularization and sterilization protocol for preparing a tissue scaffold of a human-sized liver tissue. Tissue Eng Part C Methods. 2013 Aug;19(8):642-51.

22. Nakayama KH, Lee CC, Batchelder CA, Tarantal AF. Tissue specificity of decellularized rhesus monkey kidney and lung scaffolds. PLoS One. 2013;8(5):e64134.

23. DeQuach JA, Yuan SH, Goldstein LS, Christman KL. Decellularized porcine brain matrix for cell culture and tissue engineering scaffolds. Tissue Eng Part A. 2011 Nov; 17(21-22):2583-92.

24. Sierad LN, Shaw EL, Bina A, Brazile B, Rierson N, Patnaik SS, et al. Functional Heart Valve Scaffolds Obtained by Complete Decellularization of Porcine Aortic Roots in a Novel Differential Pressure Gradient Perfusion System. Tissue Eng Part C Methods. 2015 Dec;21(12):1284-96.

25. Kerrison P, Steinke M. DAPI staining protocol.

26. Inno-train Diagnostic GMBH. Manual DNA Extraction with magnetic beads. 2019 (cited 2019); Available from: https://www.innotrain.de/en/products/dna-isolation/bexs-12ready-dna-kits/.

27. Waterbolk TW, Hoendermis ES, den Hamer IJ, Ebels T. Pulmonary valve replacement with a mechanical prosthesis. Promising results of 28 procedures in patients with congenital heart disease. Eur J Cardiothorac Surg. 2006 Jul;30(1):28-32.

28. Freling HG, van Slooten YJ, van Melle JP, Ebels T, Hoendermis ES, Berger RM, et al. Pulmonary valve replacement: twentysix years of experience with mechanical valvar prostheses. Ann Thorac Surg. 2015 Mar;99(3):905-10.

29. Schwedler G, Lindinger A, Lange PE, Sax U, Olchvary J, Peters B, et al. Frequency and spectrum of congenital heart defects among live births in Germany : a study of the Competence Network for Congenital Heart Defects. Clin Res Cardiol. 2011 Dec;100(12):1111-7.

30. Shebani SO, McGuirk S, Baghai M, Stickley J, De Giovanni JV, Bu'lock FA, et al. Right ventricular outflow tract reconstruction using Contegra valved conduit: natural history and conduit performance under pressure. Eur J Cardiothorac Surg. 2006 Mar;29(3):397405.

31. Boethig D, Horke A, Hazekamp M, Meyns B, Rega F, Van Puyvelde J, et al. A European study on decellularized homografts for pulmonary valve replacement: initial results from the prospective ESPOIR Trial and ESPOIR Registry datadagger. Eur J Cardiothorac Surg. 2019 Sep 1;56(3):503-9

32. Arise Hannover. Welcome to Arise. 2019; Available from: http://arise-clinicaltrial.eu/ home.html. 